Journal of Mathematics and Statistics 7 (1): 81-85, 2011

ISSN 1549-3644

(C) 2010 Science Publications

\title{
Half-bounded Numerical Solution of Singular Integral Equations with Cauchy Kernel
}

\author{
M. Abdulkawi, Z.K. Eshkuvatov and N.M.A. Nik Long \\ Department of Mathematics and Institute for Mathematical Research, \\ University Putra Malaysia, 43400 Serdang, Selangor, Malaysia
}

\begin{abstract}
Problem statement: In this study, a numerical solution for singular integral equations of the first kind with Cauchy kernel over the finite segment [-1,1] is presented. The numerical solution is bounded at $\mathrm{x}=1$ and unbounded at $\mathrm{x}=-1$. Approach: The numerical solution is derived by approximating the unknown density function using the weighted Chebyshev polynomials of the fourth kind. Results: The force function is approximated by using the Chebyshev polynomials of the third kind. Conclusion: The exactness of the numerical solution is shown for characteristic equation when the force function is a cubic.
\end{abstract}

Key words: Singular integral equations, Cauchy kernel, chebyshev orthogonal polynomials

\section{INTRODUCTION}

Consider the Cauchy type singular integral equations of the first kind:

$\int_{-1}^{1} \frac{\varphi(t)}{t-x} d t+\int_{-1}^{1} K(x, t) \varphi(t) d t=f(x)$

where $K(x, t)$ and $f(x)$ are assumed to be real-valued functions belong to the class of Hölder on the sets $[-1,1] \times[-1,1]$ and $[-1,1]$, respectively. $\phi(x)$ is unknown function to be determined. The theories and applications of singular integral equations are described in many references as well as in Gakhov (1990), Ladopoulos (2000), Lifanov (1996) and Nandhakumar et al. (2009).

The characteristic singular integral equation is of the form (Lifanov (1996):

$\int_{-1}^{1} \frac{\varphi(t)}{t-x} d t=f(x), \quad-1<x<1$

It is well known that the analytical solution of characteristic equation (2) which is bounded at the endpoint $x=1$ is given by the following formula:

$\phi(x)=-\frac{1}{\pi^{2}} \sqrt{\frac{1-x}{1+x}} \int_{-1}^{1} \sqrt{\frac{1+t}{1-t}} \frac{f(t)}{t-x} d t$

By solving equation (1) with respect to its characteristic part, we will find that it is equivalent to the Fredholm equation of the second kind:

$$
\begin{aligned}
& \varphi(t)+\int_{-1}^{1} \mathrm{~N}(\mathrm{t}, \tau) \varphi(\tau) \mathrm{d} \tau=\mathrm{F}(\mathrm{t}), \\
& \mathrm{N}(\mathrm{t}, \tau)=-\frac{1}{\pi^{2}} \sqrt{\frac{1-\mathrm{t}}{1+\mathrm{t}}} \int_{-1}^{1} \sqrt{\frac{1+\mathrm{x}}{1-\mathrm{x}}} \frac{\mathrm{K}(\mathrm{x}, \tau)}{\mathrm{x}-\mathrm{t}} \mathrm{dx}, \\
& \mathrm{F}(\mathrm{t})=-\frac{1}{\pi^{2}} \sqrt{\frac{1-\mathrm{t}}{1+\mathrm{t}}} \int_{-1}^{1} \sqrt{\frac{1+\mathrm{x}}{1-\mathrm{x}}} \frac{\mathrm{f}(\mathrm{x})}{\mathrm{x}-\mathrm{t}} \mathrm{dx},
\end{aligned}
$$

in the sense of obtaining the solution, which one can apply the Fredholm's theorems.

Mahiub et al. (2009) presented a numerical solution of equation (1) which is unbounded at both the endpoints $x= \pm 1$. They used Chebyshev polynomials of the first kind with the corresponding weight function to approximate the unknown density function. They proved that the numerical solution of characteristic equation is identical to the exact solution when the force function is a cubic.

Abdulkawi et al. (2009a) discussed a numerical solution of equation (1) which is bounded at the both endpoints $x= \pm 1$. They used Chebyshev polynomials of the second kind with the corresponding weight function to approximate the density function. They showed that the numerical solution of characteristic equation is identical with the exact solution when the force function is a cubic.

Abdulkawi et al. (2009b) presented an approximate solution of equation (1) which is bounded at the endpoint $x=-1$, but unbounded at the endpoint $x=1$. They used Chebyshev polynomials of the third kind with the corresponding weight function to approximate the unknown density function. They proved that the

Corresponding Author: Dr. Mohammad Abdulkawi, Department of Mathematics and Institute for Mathematical Research, University Putra Malaysia, 43400 Serdang, Selangor, Malaysia 
approximate solution of characteristic equation is identical to the exact solution when the force function is a cubic.

In this study, we present a numerical solution of equation (1) which is bounded at the endpoint $x=1$ and unbounded at the endpoint $x=-1$.

\section{MATERIALS AND METHODS}

Numerical solution: The Chebyshev polynomials of the third kind $\mathrm{V}_{\mathrm{j}}$ and fourth kind $\mathrm{W}_{\mathrm{j}}$ with the corresponding weight functions $\omega_{1}$ and $\omega_{2}$ are defined as :

$$
\left.\begin{array}{l}
\mathrm{V}_{\mathrm{i}}(\mathrm{x})=\frac{\cos \left(\frac{2 \mathrm{i}+1}{2} \theta\right)}{\cos \left(\frac{\theta}{2}\right)}, \omega_{1}(\mathrm{x})=\sqrt{\frac{1+\mathrm{x}}{1-\mathrm{x}}}, \\
\mathrm{W}_{\mathrm{i}}(\mathrm{x})=\frac{\sin \left(\frac{2 \mathrm{i}+1}{2} \theta\right)}{\sin \left(\frac{\theta}{2}\right)}, \omega_{2}(\mathrm{x})=\sqrt{\frac{1-\mathrm{x}}{1+\mathrm{x}}}, \\
\theta=\cos ^{-1} \mathrm{x} .
\end{array}\right\}
$$

It is known that (Mason and Handscomb, 2003):

$\int_{-1}^{1} \sqrt{\frac{1-\mathrm{t}}{1+\mathrm{t}}} \frac{\mathrm{W}_{\mathrm{i}}(\mathrm{t})}{\mathrm{t}-\mathrm{x}} \mathrm{dt}=-\pi \mathrm{V}_{\mathrm{i}}(\mathrm{x}),-1<\mathrm{x}<1$

Interpolating the known force function $f(x)$ as follows:

$$
\mathrm{f}(\mathrm{x}) \approx \mathrm{f}_{\mathrm{n}}(\mathrm{x})=\sum_{\mathrm{k}=0}^{\mathrm{n}} \hat{\mathrm{f}}_{\mathrm{k}} \mathrm{V}_{\mathrm{k}}(\mathrm{x})
$$

Where:

$$
\hat{\mathrm{f}}_{\mathrm{k}}=\frac{1}{\pi} \int_{-1}^{1} \sqrt{\frac{1+\mathrm{t}}{1-\mathrm{t}}} \mathrm{f}(\mathrm{t}) \mathrm{V}_{\mathrm{k}}(\mathrm{t}) \mathrm{dt}
$$

Approximating the unknown density function $\phi$ by $\phi_{\mathrm{n}}$, in which:

$\phi_{n}(x)=\sqrt{\frac{1-x}{1+x}} \sum_{j=0}^{n} b_{j} W_{j}(x)$

where the unknown coefficients $\left\{b_{j}\right\}_{0}^{n}$ are to be determined.
Substituting (9) into (1) we obtain:

$$
\begin{aligned}
& \sum_{j=0}^{n} b_{j} \int_{-1}^{1} \sqrt{\frac{1-t}{1+t}} \frac{W_{j}(t)}{t-x} d t \\
& +\sum_{j=0}^{n} b_{j} \int_{-1}^{1} \sqrt{\frac{1-t}{1+t}} K(x, t) W_{j}(t) d t=f(x)
\end{aligned}
$$

Using (6) into (10) we obtain:

$$
-\pi \sum_{j=0}^{n} b_{j} V_{j}(x)+\sum_{j=0}^{n} b_{j} \eta_{j}(x)=f(x)
$$

where:

$$
\eta_{j}(x)=\int_{-1}^{1} \sqrt{\frac{1-t}{1+t}} K(x, t) W_{j}(t) d t
$$

Now, we approximate the function $\eta_{j}(x)$ as:

$$
\eta_{\mathrm{j}}(\mathrm{x}) \approx \sum_{\mathrm{k}=0}^{\mathrm{n}} \lambda_{\mathrm{jk}} \mathrm{V}_{\mathrm{k}}(\mathrm{x})
$$

Where:

$$
\lambda_{\mathrm{jk}}=\frac{1}{\pi} \int_{-1}^{1} \omega_{1}(\mathrm{x}) \int_{-1}^{1} \omega_{2}(\mathrm{t}) \mathrm{K}(\mathrm{x}, \mathrm{t}) \mathrm{W}_{\mathrm{j}}(\mathrm{t}) \mathrm{V}_{\mathrm{k}}(\mathrm{x}) \mathrm{dtdx}
$$

Due to (7) and (13), equation (11) becomes:

$\sum_{j=0}^{n} b_{j} V_{j}(x)-\frac{1}{\pi} \sum_{k=0}^{n} \sum_{j=0}^{n} b_{j} \lambda_{j k} V_{k}(x)=-\frac{1}{\pi} \sum_{k=0}^{n} \hat{f}_{k} V_{k}(x)$

The unknown coefficients $\left\{b_{j}\right\}_{0}^{n}$ are determined by solving the following system of linear equations obtained by comparing the coefficients of $\mathrm{V}_{\mathrm{j}}, \mathrm{j}=0,1,2, \ldots, \mathrm{n}$ in both sides of equation (15):

$$
\begin{array}{ll}
\mathrm{b}_{0}-\frac{1}{\pi} \sum_{j=0}^{\mathrm{n}} \mathrm{b}_{\mathrm{j}} \lambda_{\mathrm{j} 0} & =-\frac{1}{\pi} \hat{\mathrm{f}}_{0}, \\
\mathrm{~b}_{1}-\frac{1}{\pi} \sum_{j=0}^{n} \mathrm{~b}_{\mathrm{j}} \lambda_{\mathrm{j} 1} & =-\frac{1}{\pi} \hat{\mathrm{f}}_{1}, \\
\vdots \quad \vdots \quad & \vdots \\
\mathrm{b}_{\mathrm{n}}-\frac{1}{\pi} \sum_{j=0}^{\mathrm{n}} \mathrm{a}_{\mathrm{j}} \lambda_{\mathrm{j}} & =-\frac{1}{\pi} \hat{\mathrm{f}}_{\mathrm{n}},
\end{array}
$$

where the coefficients $\hat{\mathrm{f}}_{\mathrm{i}}$ and $\lambda_{\mathrm{jk}}$ are given by (8) and (14), respectively. 
Exactness of the numerical solution:

Proposition 1. If $f(x)$ in equation (2) is a cubic function, then the approximate solution (9) of characteristic equation (2) is exact.

Proof: Let $f(x)$ in equation (2) be a cubic function, i.e:

$\mathrm{f}(\mathrm{x})=\mathrm{c}_{0}+\mathrm{c}_{1} \mathrm{x}+\mathrm{c}_{2} \mathrm{x}^{2}+\mathrm{c}_{3} \mathrm{x}^{3}$

Substituting (17) into (2), we have:

$\int_{-1}^{1} \frac{\phi(t)}{t-x} d t=c_{0}+c_{1} x+c_{2} x^{2}+c_{3} x^{3},-1<x<1$

Substitution (17) into (8) yields:

$\hat{\mathrm{f}}_{\mathrm{k}}=\frac{1}{\pi} \int_{-1}^{1} \sqrt{\frac{1+\mathrm{t}}{1-\mathrm{t}}}\left(\mathrm{c}_{0}+\mathrm{c}_{1} \mathrm{t}+\mathrm{c}_{2} \mathrm{t}^{2}+\mathrm{c}_{3} \mathrm{t}^{3}\right) \mathrm{V}_{\mathrm{k}}(\mathrm{t}) \mathrm{dt}$

The Chebyshev recurrence relations of the third and fourth kinds, respectively, are:

$\mathrm{V}_{0}(\mathrm{x})=1, \mathrm{~V}_{1}(\mathrm{x})=2 \mathrm{x}-1$,

$\left.V_{n}(x)=2 x V_{n-1}(x)-V_{n-2}(x), n \geq 2, \quad\right\}$

and:

$\mathrm{W}_{0}(\mathrm{x})=1, \mathrm{~W}_{1}(\mathrm{x})=2 \mathrm{x}+1$,

$\mathrm{W}_{\mathrm{n}}(\mathrm{x})=2 \mathrm{xW}_{\mathrm{n}-1}(\mathrm{x})-\mathrm{W}_{\mathrm{n}-2}(\mathrm{x}), \mathrm{n} \geq 2 . \quad$

With help of (20) and (21), we have:

$$
\begin{aligned}
t^{3} & =\frac{1}{8}\left[\mathrm{~V}_{3}(\mathrm{t})+\mathrm{V}_{2}(\mathrm{t})+3\left(\mathrm{~V}_{1}(\mathrm{t})+\mathrm{V}_{0}(\mathrm{t})\right)\right] \\
= & \frac{1}{8}\left[\mathrm{~W}_{3}(\mathrm{t})-\mathrm{W}_{2}(\mathrm{t})+3\left(\mathrm{~W}_{1}(\mathrm{t})-\mathrm{W}_{0}(\mathrm{t})\right)\right] \\
\mathrm{t}^{2} & =\frac{1}{4}\left[\mathrm{~V}_{2}(\mathrm{t})+\mathrm{V}_{1}(\mathrm{t})+2 \mathrm{~V}_{0}(\mathrm{t})\right] \\
& =\frac{1}{4}\left[\mathrm{~W}_{2}(\mathrm{t})-\mathrm{W}_{1}(\mathrm{t})+2 \mathrm{~W}_{0}(\mathrm{t})\right] \\
\mathrm{t} & =\frac{1}{2}\left[\mathrm{~V}_{1}(\mathrm{t})+\mathrm{V}_{0}(\mathrm{t})\right] \\
& =\frac{1}{2}\left[\mathrm{~W}_{1}(\mathrm{t})-\mathrm{W}_{0}(\mathrm{t})\right]
\end{aligned}
$$

It is known that the Chebyshev polynomials of the third and fourth kinds are orthogonals with respect to the weight functions $\omega_{1}$ and $\omega_{2}$, respectively, i.e.:

$\int_{-1}^{1} \sqrt{\frac{1+\mathrm{t}}{1-\mathrm{t}}} \mathrm{V}_{\mathrm{m}}(\mathrm{t}) \mathrm{V}_{\mathrm{n}}(\mathrm{t}) \mathrm{dt}= \begin{cases}0, & \mathrm{n} \neq \mathrm{m}, \\ \pi, & \mathrm{n}=\mathrm{m},\end{cases}$ and:

$$
\int_{-1}^{1} \sqrt{\frac{1-\mathrm{t}}{1+\mathrm{t}}} \mathrm{W}_{\mathrm{m}}(\mathrm{t}) \mathrm{W}_{\mathrm{n}}(\mathrm{t}) \mathrm{dt}= \begin{cases}0, & \mathrm{n} \neq \mathrm{m}, \\ \pi, & \mathrm{n}=\mathrm{m} .\end{cases}
$$

Taking into account (22) and using the orthogonal property (23) into (19), we obtain:

$$
\left.\begin{array}{l}
\hat{\mathrm{f}}_{0}=\mathrm{c}_{0}+\frac{\mathrm{c}_{1}+\mathrm{c}_{2}}{2}+\frac{3 \mathrm{c}_{3}}{8}, \\
\hat{\mathrm{f}}_{1}=\frac{2 \mathrm{c}_{1}+\mathrm{c}_{2}}{4}+\frac{3 \mathrm{c}_{3}}{8}, \\
\hat{\mathrm{f}}_{2}=\frac{2 \mathrm{c}_{2}+\mathrm{c}_{3}}{8}, \\
\hat{\mathrm{f}}_{3}=\frac{\mathrm{c}_{3}}{8} .
\end{array}\right\}
$$

From the system $(16)$ where $\mathrm{K}(\mathrm{x}, \mathrm{t})=0$ yields:

$b_{j}=-\frac{1}{\pi} \hat{f}_{j}, \quad j=0, \ldots, n$

Substituting (26) into (9) for $n=3$, we get:

$$
\begin{aligned}
\varphi_{\mathrm{n}}(\mathrm{x})=-\frac{1}{\pi} \sqrt{\frac{1-\mathrm{x}}{1+\mathrm{x}}} \times \\
\quad\left[\hat{\mathrm{f}}_{0}+\hat{\mathrm{f}}_{1} \mathrm{~W}_{1}(\mathrm{x})+\hat{\mathrm{f}}_{2} \mathrm{~W}_{2}(\mathrm{x})+\hat{\mathrm{f}}_{3} \mathrm{~W}_{3}(\mathrm{x})\right]
\end{aligned}
$$

Thus the approximate solution of equation (18) is:

$$
\left.\begin{array}{l}
\phi_{\mathrm{n}}(\mathrm{x})=-\frac{1}{\pi} \sqrt{\frac{1-\mathrm{x}}{1+\mathrm{x}}} \mathrm{p}(\mathrm{x}), \\
\mathrm{p}(\mathrm{x})=\mathrm{c}_{0}+\mathrm{c}_{1}+\frac{1}{2}\left(\mathrm{c}_{2}+\mathrm{c}_{3}\right) \\
+\left(\mathrm{c}_{1}+\mathrm{c}_{2}+\frac{1}{2} \mathrm{c}_{3}\right) \mathrm{x}+\left(\mathrm{c}_{2}+\mathrm{c}_{3}\right) \mathrm{x}^{2}+\mathrm{c}_{3} \mathrm{x}^{3} .
\end{array}\right\}
$$

In order to obtain the exact solution of equation (18), we substitute (17) into (3) which gives:

$$
\begin{aligned}
\varphi(x)= & -\frac{1}{\pi^{2}} \sqrt{\frac{1-\mathrm{x}}{1+\mathrm{x}}} \mathrm{x} \\
& \int_{-1}^{1} \sqrt{\frac{1+\mathrm{t}}{1-\mathrm{t}}} \frac{\mathrm{c}_{0}+\mathrm{c}_{1} \mathrm{t}+\mathrm{c}_{2} \mathrm{t}^{2}+\mathrm{c}_{3} \mathrm{t}^{3}}{\mathrm{t}-\mathrm{x}} \mathrm{dt}
\end{aligned}
$$

It is easy to see that:

$\int_{-1}^{1} \sqrt{\frac{1+\mathrm{t}}{1-\mathrm{t}}} \frac{1}{\mathrm{t}-\mathrm{x}} \mathrm{dt}=\pi$,

$\int_{-1}^{1} \sqrt{\frac{1+\mathrm{t}}{1-\mathrm{t}}} \frac{\mathrm{t}}{\mathrm{t}-\mathrm{x}} \mathrm{dt}=\pi(1+\mathrm{x})$

$\int_{-1}^{1} \sqrt{\frac{1+\mathrm{t}}{1-\mathrm{t}}} \frac{\mathrm{t}^{2}}{\mathrm{t}-\mathrm{x}} \mathrm{dt}=\pi\left(0.5+\mathrm{x}+\mathrm{x}^{2}\right)$,

$\int_{-1}^{1} \sqrt{\frac{1+\mathrm{t}}{1-\mathrm{t}}} \frac{\mathrm{t}^{3}}{\mathrm{t}-\mathrm{x}} \mathrm{dt}=\pi\left(0.5+0.5 \mathrm{x}+\mathrm{x}^{2}+\mathrm{x}^{3}\right)$. 
From (29)-(30) we obtain the exact solution of equation (18) which is identical to the approximate solution given by (28). The proof is complete.

\section{RESULTS AND DISCUSSION}

In this section, we discuss a particular example to show the accuracy of our numerical solution.

Let us consider the integral equation (1) with degenerate kernel $k(x, t)=x^{5} t^{5}$ and polynomial function $f(x)=x^{5}+x^{3}+x$, i.e.:

$$
\int_{-1}^{1} \frac{\varphi(t)}{t-x} d t+\int_{-1}^{1} x^{5} t^{5} \varphi(t) d t=x^{5}+x^{3}+x
$$

and we seek the solution of this equation, which is bounded at the endpoint $x=1$ and unbounded at the endpoint $x=-1$.

Due to (8), we have:

$$
\hat{\mathrm{f}}_{\mathrm{k}}=\frac{1}{\pi} \int_{-1}^{1} \sqrt{\frac{1+\mathrm{t}}{1-\mathrm{t}}}\left(\mathrm{t}^{5}+\mathrm{t}^{3}+\mathrm{t}\right) \mathrm{V}_{\mathrm{k}}(\mathrm{t}) \mathrm{dt}
$$

It is easy to see that:

$$
\left.\begin{array}{rl}
\mathrm{t}^{5}= & \frac{1}{32}\left[\mathrm{~V}_{5}(\mathrm{t})+\mathrm{V}_{4}(\mathrm{t})+5\left(\mathrm{~V}_{3}(\mathrm{t})+\mathrm{V}_{2}(\mathrm{t})\right)\right. \\
& \left.+10\left(\mathrm{~V}_{1}(\mathrm{t})+\mathrm{V}_{0}(\mathrm{t})\right)\right] \\
= & \frac{1}{32}\left[\mathrm{~W}_{5}(\mathrm{t})-\mathrm{W}_{4}(\mathrm{t})+5\left(\mathrm{~W}_{3}(\mathrm{t})-\mathrm{W}_{2}(\mathrm{t})\right)\right. \\
& \left.+10\left(\mathrm{~W}_{1}(\mathrm{t})-\mathrm{V}_{0}(\mathrm{t})\right)\right]
\end{array}\right\}
$$

From (22), (32) and (33) we obtain:

$$
\hat{\mathrm{f}}_{0}=\hat{\mathrm{f}}_{1}=\frac{19}{16}, \hat{\mathrm{f}}_{2}=\hat{\mathrm{f}}_{3}=\frac{9}{32}, \hat{\mathrm{f}}_{4}=\hat{\mathrm{f}}_{5}=\frac{1}{32}
$$

From (14) where $K(x, t)=x^{5} t^{5}$, we have:

$$
\begin{aligned}
\lambda_{j k}= & \frac{1}{\pi} \int_{-1}^{1} \sqrt{\frac{1+x}{1-x}} x^{5} \int_{-1}^{1} \sqrt{\frac{1-t}{1+t}} t^{5} W_{j}(t) V_{k}(x) d t d x \\
= & \frac{1}{\pi}\left[\int_{-1}^{1} \sqrt{\frac{1+x}{1-x}} x^{5} V_{k}(x) d x\right] \\
& \times\left[\int_{-1}^{1} \sqrt{\frac{1-t}{1+t}} t^{5} W_{j}(t) d t\right]
\end{aligned}
$$

Due to (23), (24) and (33), yields:

$$
\begin{aligned}
& \lambda_{00}=\lambda_{01}=-\frac{100 \pi}{(32)^{2}}, \lambda_{02}=\lambda_{03}=-\frac{50 \pi}{(32)^{2}}, \\
& \lambda_{04}=\lambda_{05}=-\frac{10 \pi}{(32)^{2}}, \lambda_{10}=\lambda_{11}=\frac{100 \pi}{(32)^{2}}, \\
& \lambda_{12}=\lambda_{13}=\frac{50 \pi}{(32)^{2}}, \lambda_{14}=\lambda_{15}=\frac{10 \pi}{(32)^{2}}, \\
& \lambda_{20}=\lambda_{21}=-\frac{50 \pi}{(32)^{2}}, \lambda_{22}=\lambda_{23}=-\frac{25 \pi}{(32)^{2}}, \\
& \lambda_{24}=\lambda_{25}=-\frac{5 \pi}{(32)^{2}}, \lambda_{30}=\lambda_{31}=\frac{50 \pi}{(32)^{2}}, \\
& \lambda_{32}=\lambda_{33}=\frac{25 \pi}{(32)^{2}}, \lambda_{34}=\lambda_{35}=\frac{5 \pi}{(32)^{2}}, \\
& \lambda_{40}=\lambda_{41}=-\frac{10 \pi}{(32)^{2}}, \lambda_{42}=\lambda_{43}=-\frac{5 \pi}{(32)^{2}}, \\
& \lambda_{44}=\lambda_{4,5}=-\frac{\pi}{(32)^{2}}, \lambda_{50}=\lambda_{51}=\frac{10 \pi}{(32)^{2}}, \\
& \lambda_{52}=\lambda_{53}=\frac{5 \pi}{(32)^{2}}, \lambda_{54}=\lambda_{55}=\frac{\pi}{(32)^{2}} .
\end{aligned}
$$

Then the unknown coefficients $b_{j}, j=0, \ldots, 5$ are obtained by solving the following system of linear equations:

$$
\left.\begin{array}{c}
\mathrm{b}_{0}+\frac{100}{(32)^{2}} \mathrm{~b}_{0}-\frac{100}{(32)^{2}} \mathrm{~b}_{1}+\frac{50}{(32)^{2}} \mathrm{~b}_{2} \\
-\frac{50}{(32)^{2}} \mathrm{~b}_{3}+\frac{10}{(32)^{2}} \mathrm{~b}_{4}-\frac{10}{(32)^{2}} \mathrm{~b}_{5}=\frac{-19}{16 \pi}, \\
\mathrm{b}_{1}+\frac{100}{(32)^{2}} \mathrm{~b}_{0}-\frac{100}{(32)^{2}} \mathrm{~b}_{1}+\frac{50}{(32)^{2}} \mathrm{~b}_{2} \\
-\frac{50}{(32)^{2}} \mathrm{~b}_{3}+\frac{10}{(32)^{2}} \mathrm{~b}_{4}-\frac{10}{(32)^{2}} \mathrm{~b}_{5}=\frac{-19}{16 \pi}, \\
\mathrm{b}_{2}+\frac{50}{(32)^{2}} \mathrm{~b}_{0}-\frac{50}{(32)^{2}} \mathrm{~b}_{1}+\frac{25}{(32)^{2}} \mathrm{~b}_{2} \\
-\frac{25}{(32)^{2}} \mathrm{~b}_{3}+\frac{5}{(32)^{2}} \mathrm{~b}_{4}-\frac{5}{(32)^{2}} \mathrm{~b}_{5}=\frac{-9}{32 \pi}, \\
\mathrm{b}_{3}+\frac{50}{(32)^{2}} \mathrm{~b}_{0}-\frac{50}{(32)^{2}} \mathrm{~b}_{1}+\frac{25}{(32)^{2}} \mathrm{~b}_{2} \\
-\frac{25}{(32)^{2}} \mathrm{~b}_{3}+\frac{5}{(32)^{2}} \mathrm{~b}_{4}-\frac{5}{(32)^{2}} \mathrm{~b}_{5}=\frac{-9}{32 \pi}, \\
\mathrm{b}_{4}+\frac{10}{(32)^{2}} \mathrm{~b}_{0}-\frac{10}{(32)^{2}} \mathrm{~b}_{1}+\frac{5}{(32)^{2}} \mathrm{~b}_{2} \\
-\frac{5}{(32)^{2}} \mathrm{~b}_{3}+\frac{1}{(32)^{2}} \mathrm{~b}_{4}-\frac{1}{(32)^{2}} \mathrm{~b}_{5}=\frac{-1}{32 \pi}, \\
\mathrm{b}_{5}+\frac{10}{(32)^{2}} \mathrm{~b}_{0}-\frac{10}{(32)^{2}} \mathrm{~b}_{1}+\frac{5}{(32)^{2}} \mathrm{~b}_{2} \\
-\frac{5}{(32)^{2}} \mathrm{~b}_{3}+\frac{1}{(32)^{2}} \mathrm{~b}_{4}-\frac{1}{(32)^{2}} \mathrm{~b}_{5}=\frac{-1}{32 \pi},
\end{array}\right\}
$$

which are: 


$$
\left.\begin{array}{l}
\mathrm{b}_{0}=\mathrm{b}_{1}=-\frac{19}{16 \pi}, \mathrm{b}_{2}=\mathrm{b}_{3}=-\frac{9}{32 \pi}, \\
\mathrm{b}_{4}=\mathrm{b}_{5}=-\frac{1}{32 \pi} .
\end{array}\right\}
$$

Substituting the values of the coefficients $\left\{b_{j}\right\}_{0}^{5}$ into (9) yields the approximate solution of equation (31), which is:

$$
\begin{aligned}
\varphi_{\mathrm{n}}(\mathrm{x})= & -\frac{1}{\pi} \sqrt{\frac{1-\mathrm{x}}{1+\mathrm{x}}} \times \\
& {\left[\mathrm{x}^{5}+\mathrm{x}^{4}+\frac{3}{2}\left(\mathrm{x}^{3}+\mathrm{x}^{2}\right)+\frac{15}{8}(\mathrm{x}+1)\right] }
\end{aligned}
$$

It is not difficult to see that the approximate solution (39) is exact.

\section{CONCLUSION}

The Chebyshev orthogonal polynomials of the third and fourth kinds are used to approximate the known force function and unknown density function, respectively. Proposition 1 shows the exactness of the numerical solution for characteristic equation when the force function is a cubic. Particular results show that our numerical solution does not only give the exact solution for characteristic equation but also for other Cauchy singular integral equations.

\section{ACKNOWLEDGEMENT}

This study was supported by University Putra Malaysia under project No. 05-03-10-0988RU.

\section{REFERENCES}

Abdulkawi, M., Z.K. Eshkuvatov and N.M.A.N. Long, 2009a. A note on the numerical solution of singular integral equations of cauchy type. Int. J. Math. Comput. Sci., 5: 90-93.

Abdulkawi, M., Z.K. Eshkuvatov and N.M.A.N. Long, 2009b. On the semi-bounded solution of Cauchy type singular integral equations of the first kind. European J. Pure nd Applied Math., 2: 462-472.

Gakhov, F.D., 1990. Boundary value problems. 1st Edn., Dover Publications, USA., ISBN-10: 0486662756, pp: 581.

Ladopoulos, E.G., 2000. Singular integral equations: Linear and Non-Linear, Theory and its applications in Science and Engineering. 1st Edn., Springer, USA., ISBN-10: 3540672303, pp: 584.

Lifanov, I.K., 1996. Singular Integral Equations and Discrete Vortices. 1st Edn., Walter de Gruyter, The Netherlands, ISBN-10: 906764207X, pp: 475.

Mahiub, M.A., Z.K. Eshkuvatov and N.M.A. N. Long, 2009. A method for the numerical solution of cauchy singular integral equations. Int. J. Applied Math., 22: 287-297.

Mason, J.C. and D.C. Handscomb, 2003. Chebyshev polynomials. 1st Edn., Chapman and Hall/CRC, USA., ISBN-10: 0849303559, pp: 341.

Nandhakumar, S., V. Selladurai and S. Sekar, 2009. Numerical investigation of an industrial robot arm control problem using haar wavelet series. Am. J. Eng. Applied Sci., 2: 584-589. DOI: 10.3844/ajeassp.2009.584.589 